\title{
COMPACT HERMITIAN OPERATORS ON PROJECTIVE TENSOR PRODUCTS OF BANACH ALGEBRAS
}

\author{
T. K. DUTTA, H. K. NATH, and H. K. SARMAH
}

Received 24 February 2000

\begin{abstract}
Let $U$ and $V$ be, respectively, an infinite- and a finite-dimensional complex Banach algebras, and let $U \otimes_{p} V$ be their projective tensor product. We prove that (i) every compact Hermitian operator $T_{1}$ on $U$ gives rise to a compact Hermitian operator $T$ on $U \otimes p V$ having the properties that $\left\|T_{1}\right\|=\|T\|$ and $\operatorname{sp}\left(T_{1}\right)=\operatorname{sp}(T)$; (ii) if $U$ and $V$ are separable and $U$ has Hermitian approximation property (HAP), then $U \otimes_{p} V$ is also separable and has HAP; (iii) every compact analytic semigroup (CAS) on $U$ induces the existence of a CAS on $U \otimes_{p} V$ having some nice properties. In addition, the converse of the above results are discussed and some open problems are posed.
\end{abstract}

2000 Mathematics Subject Classification: 46M05, 47B07, 47B15.

1. Introduction. We first introduce the terminologies which are initially needed for our main purpose.

1.1. The projective tensor norm $\|\cdot\|_{p}$ is defined on the algebraic tensor product $U \otimes V$ of two complex Banach algebras $U$ and $V$ by

$$
\|a\|_{p}=\inf \left\{\sum_{i=1}^{n}\left\|u_{i}\right\|\left\|v_{i}\right\|: a=\sum_{i=1}^{n} u_{i} \otimes v_{i}\right\},
$$

where the infimum is taken over all (finite) representations of $a \in U \otimes V$. The projective tensor product $U \otimes_{p} V$ is the completion of $U \otimes V$ with this norm. Furthermore, a norm $\|\cdot\|_{\pi}$ on $U \otimes V$ is said to be a cross-norm if $\|u \otimes v\|_{\pi}=\|u\|\|v\|$. (For detailed discussion of various tensor products, see $[1,3,6,7]$.)

1.2. A state on a unital Banach algebra $U$ with the unit $e$ is a continuous linear functional $f$ such that $\|f\|=f(e)=1$, and an element $u$ in $U$ is Hermitian if and only if its numerical range, that is, $N(u)=\{f(u): f$ is a state on $U\}$, is contained in the real line. Equivalently, $u$ is Hermitian if and only if $\lim _{\alpha \rightarrow 0+}(1 / \alpha)[\|e+i \alpha u\|-1]=0$. If an operator $T$ on $U$ is such that it is a Hermitian as an element of the operator algebra, then $T$ is called a Hermitian operator on $U$.

1.3. A linear transformation $T$ mapping a normed linear space $X$ into a normed linear space $Y$ is said to be compact if, given any sequence $\left\{x_{n}\right\}$ in $X$ such that $\left\{\left\|x_{n}\right\|\right\}$ is bounded, the sequence $\left\{T x_{n}\right\}$ has a convergent subsequence. If $T$ is both compact and Hermitian, it is then termed as a compact Hermitian operator. A compact Hermitian operator enjoys many powerful technical results (see [4]). 
Throughout this paper, unless stated specifically, $U$ denotes an infinite-dimensional complex Banach algebra with its dual $U^{*}, V$ is a finite-dimensional complex Banach algebra with $\operatorname{dim} V=k,\left\{e_{1}, e_{2}, \ldots, e_{k}\right\}$ a standardized basis for $V$ with $\left\|e_{j}\right\|=1$, for all $j=1,2, \ldots, k$ and $U \otimes_{p} V$ their projective tensor product. Our primary aim is to study how a compact Hermitian operator on $U$, the Hermitian approximation property in $U$ and a compact analytic semigroup on $U$ gives rise to the significant existence of those on $U \otimes_{p} V$. The converse route from $U \otimes_{p} V$ to $U$ with these properties is also investigated with some fruitful outcomes.

2. The main results. We first prove a simple but illuminating lemma.

LEMMA 2.1. Every element a of $U \otimes_{p} V$ can be expressed in the form $\sum_{j=1}^{k} w_{j} \otimes e_{j}$.

Proof. We know that $U \otimes_{p} V$ is a Banach algebra consisting of all elements of the form

$$
a=\sum_{i=1}^{\infty} u_{i} \otimes v_{i}, \quad \text { where } \sum_{i=1}^{\infty}\left\|u_{i}\right\|\left\|v_{i}\right\|<\infty,
$$

(see [5]). We can choose $v_{i}$ such that $\left\|v_{i}\right\|=1$ for all $i$. If $\left\|v_{i}\right\| \neq 1$, put $v_{i}^{\prime}=v_{i} /\left\|v_{i}\right\|$ and replace $v_{i}$ by $\left\|v_{i}\right\| v_{i}^{\prime}$ and adjoin $\left\|v_{i}\right\|$ to $u_{i}$. Then $v_{i}=\sum_{j=1}^{k} \alpha_{i j} e_{j}$, where $\alpha_{i j}$ 's are scalars.

Now

$$
\begin{aligned}
a_{n} & =\sum_{i=1}^{n} u_{i} \otimes v_{i}=\sum_{i=1}^{n} u_{i} \otimes \sum_{j=1}^{k} \alpha_{i j} e_{j} \\
& =\sum_{j=1}^{k}\left(\sum_{i=1}^{n} \alpha_{i j} u_{i}\right) \otimes e_{j}=\sum_{j=1}^{k} w_{j, n} \otimes e_{j}, \quad \text { where } w_{j, n}=\sum_{i=1}^{n} \alpha_{i j} u_{i} \in U .
\end{aligned}
$$

If $v=\sum_{j=1}^{k} \beta_{j} e_{j}$ is an arbitrary element of $V$, then the map $\phi: V \rightarrow \ell_{k}^{\infty}$, defined by $\phi(v)=\left(\beta_{1}, \beta_{2}, \ldots, \beta_{k}\right)$, is a topological isomorphism. Hence there is a positive constant $M$ such that $\|\phi(v)\| \leq M\|v\|$, for all $v \in V$. Substituting $v_{i}$ in $v,\left\|\phi\left(v_{i}\right)\right\| \leq$ $M\left\|v_{i}\right\|=M$, for all $i$. This gives

$$
\operatorname{Max}_{1 \leq j \leq k}\left\{\left|\alpha_{i j}\right|\right\} \leq M
$$

Also,

$$
\sum_{i=1}^{n}\left|\alpha_{i j}\right|\left\|u_{i}\right\| \leq M \sum_{i=1}^{n}\left\|u_{i}\right\| \leq M \sum_{i=1}^{\infty}\left\|u_{i}\right\|<\infty .
$$

This shows that the partial sums of the series $\sum_{i=1}^{\infty} \alpha_{i j} u_{i}$ are absolutely uniformly bounded and hence the series $\sum_{i=1}^{\infty} \alpha_{i j} u_{i}$ is absolutely summable. Consequently, $\sum_{i=1}^{\infty} \alpha_{i j} u_{i}$ is summable to an element, say $w_{j}$ in $U$. Thus, $\lim _{n \rightarrow \infty} w_{j, n}=w_{j}$ and so $a=\lim _{n \rightarrow \infty} a_{n}=\sum_{j=1}^{k} w_{j} \otimes e_{j}$.

Next, our first key result is the following.

THEOREM 2.2. Every compact Hermitian operator $T_{1}$ on $U$ gives rise to a compact Hermitian operator $T$ on $U \otimes_{p} V$ having the properties that $\left\|T_{1}\right\|=\|T\|$ and $\operatorname{sp}\left(T_{1}\right)=$ $\operatorname{sp}(T)$. 
Proof. We prove the result in different steps.

STEP 1. One can define a map $T: U \otimes_{p} V \rightarrow U \otimes_{p} V$ by the rule

$$
T\left(\sum_{i} u_{i} \otimes v_{i}\right)=\sum_{i}\left(T_{1} u_{i}\right) \otimes v_{i} \quad \forall a=\sum_{i} u_{i} \otimes v_{i} \in U \otimes_{p} V .
$$

It is easy to show that the map is well defined. Moreover, the linearity of $T$ follows immediately from its definition.

Next we aim at proving the bound of $T$. For any arbitrary element $a \in U \otimes_{p} V$ and $\varepsilon>0$, the definition of the projective norm provides a finite representation $\sum_{i=1}^{n} u_{i} \otimes v_{i}$ such that

$$
\|a\|_{p}+\varepsilon>\sum_{i=1}^{n}\left\|u_{i}\right\|\left\|v_{i}\right\|
$$

For this representation of $a$ we obtain

$$
\|T a\|_{p}=\left\|\sum_{i=1}^{n}\left(T_{1} u_{i}\right) \otimes v_{i}\right\|_{p} \leq\left\|T_{1}\right\| \sum_{i=1}^{n}\left\|u_{i}\right\|\left\|v_{i}\right\|<\left\|T_{1}\right\|\left(\|a\|_{p}+\varepsilon\right) .
$$

Since $\varepsilon$ is arbitrary, it follows that $\left\|T_{a}\right\|_{p} \leq\left\|T_{1}\right\|\|a\|_{p}$ for each $a \in U \otimes_{p} V$.

Consequently, $T$ is bounded. Furthermore, the compactness of $T$ can be proved without much difficulty.

STEP 2. Our next attempt is to show that $T$ is Hermitian. $T_{1}$ is given to be Hermitian and therefore $\lim _{\alpha \rightarrow 0+}(1 / \alpha)\left\{\left\|I_{1}+i \alpha T_{1}\right\|-1\right\}=0$, where $\alpha$ is real and $I_{1}$ is the identity map on $U$. Let $I$ be the identity map on $U \otimes_{p} V, \varepsilon>0$, and let $\sum_{i=1}^{n} u_{i} \otimes v_{i}$ be a finite representation of $a$ such that $\|a\|_{p}+\varepsilon>\sum_{i=1}^{n}\left\|u_{i}\right\|\left\|v_{i}\right\|$. Then we can obtain

$$
\|(I+i \alpha T) a\|_{p} \leq\left\|I_{1}+i \alpha T_{1}\right\|\|a\|_{p} \quad \forall a \in U \otimes_{p} V .
$$

This gives $\|(I+i \alpha T)\| \leq\left\|I_{1}+i \alpha T_{1}\right\|$.

On the other hand, let $u \in U$ with $\|u\|=1$. Choose $v \in V$ such that $\|v\|=1$. Then $\|u \otimes v\|_{p}=1$.

Now,

$$
\begin{aligned}
\|(I+i \alpha T)\| & =\sup \left\{\|(I+i \alpha T) a\|_{p}:\|a\|_{p}=1\right\} \\
& \geq\|(I+i \alpha T)(u \otimes v)\|_{p} \\
& =\left\|u \otimes v+\left(i \alpha T_{1} u\right) \otimes v\right\|_{p} \\
& =\left\|\left[\left(I_{1}+i \alpha T_{1}\right) u\right] \otimes v\right\|_{p} \\
& =\left\|\left(I_{1}+i \alpha T_{1}\right) u\right\|\|v\| \\
& =\left\|\left(I_{1}+i \alpha T_{1}\right) u\right\| .
\end{aligned}
$$

Thus, $\|I+i \alpha T\| \geq\left\|\left(I_{1}+i \alpha T_{1}\right) u\right\|$, for all $u \in U$ with $\|u\|=1$. This yields $\|I+i \alpha T\| \geq$ $\left\|I_{1}+i \alpha T_{1}\right\|$ and so $\|I+i \alpha T\|=\left\|I_{1}+i \alpha T_{1}\right\|$.

Therefore, $\lim _{\alpha \rightarrow 0+}((\|I+i \alpha T\|-1) / \alpha)=\lim _{\alpha \rightarrow 0+}\left(\left(\left\|I_{1}+i \alpha T_{1}\right\|-1\right) / \alpha\right)=0$ and hence $T$ is Hermitian. 
STEP 3. From Step 1 we can obtain that $\|T a\|_{p} \leq\left\|T_{1}\right\|\|a\|_{p}$, for each $a \in U \otimes_{p} V$. This implies that $\|T\| \leq\left\|T_{1}\right\|$. The converse inequality $\left\|T_{1}\right\| \leq\|T\|$ can also be established without much difficulty. This establishes that $\|T\|=\left\|T_{1}\right\|$.

STEP 4. We now concentrate on the result $\operatorname{sp}\left(T_{1}\right)=\operatorname{sp}(T)$.

Let

$$
\begin{aligned}
\lambda_{1} \in \operatorname{sp}\left(T_{1}\right) & \Longrightarrow T_{1}-\lambda_{1} I_{1} \text { is singular } \\
& \Longrightarrow \exists \text { a nonzero vector } u \in U \text { such that }\left(T_{1}-\lambda_{1} I_{1}\right) u=0 .
\end{aligned}
$$

Let $v \in V$ be a vector with $v \neq 0$. Then $u \otimes v \in U \otimes_{p} V$ with $u \otimes v \neq 0$.

Now,

$$
\begin{aligned}
\left(T-\lambda_{1} I\right)(u \otimes v) & =T(u \otimes v)-\lambda_{1} I(u \otimes v) \\
& =\left(T_{1} u\right) \otimes v-\left(\lambda_{1} u\right) \otimes v \\
& =\left(T_{1}-\lambda_{1} I_{1}\right) u \otimes v=0 .
\end{aligned}
$$

Consequently, $\lambda_{1} \in \operatorname{sp}(T)$ and thus $\operatorname{sp}\left(T_{1}\right) \subseteq \operatorname{sp}(T)$. On the other hand, let $\lambda \in \operatorname{sp}(T)$. Then there exists a nonzero vector $a=\sum_{j=1}^{k} u_{j} \otimes e_{j} \in U \otimes_{p} V$, such that

$$
\begin{aligned}
(T-\lambda I) a=0 & \Rightarrow T\left(\sum_{j=1}^{k} u_{j} \otimes e_{j}\right)-\lambda \sum_{j=1}^{k} u_{j} \otimes e_{j}=0 \\
& \Longrightarrow \sum_{j=1}^{k}\left(T_{1} u_{j}\right) \otimes e_{j}-\sum_{j=1}^{k}\left(\lambda u_{j}\right) \otimes e_{j}=0 \\
& \Longrightarrow \sum_{j=1}^{k}\left(T_{1}-\lambda I_{1}\right) u_{j} \otimes e_{j}=0 \\
& \Longrightarrow\left(T_{1}-\lambda I_{1}\right) u_{j}=0 \quad \forall j=1,2, \ldots, k .
\end{aligned}
$$

Since $u \neq 0$, there exists at least one $j$ such that $u_{j} \neq 0$. Hence, $\lambda$ is an eigenvalue of $T_{1}$. So, $\operatorname{sp}(T) \subseteq \operatorname{sp}\left(T_{1}\right)$. Ultimately we have $\operatorname{sp}(T)=\operatorname{sp}\left(T_{1}\right)$.

REMARK 2.3. (i) If both $U$ and $V$ are infinite-dimensional Banach algebras, then the compactness of $T_{1}$ may not imply that of $T$. For example, choose a sequence $\left\{v_{n}\right\}$ in $V$ with $\left\|v_{n}\right\|=1$ so that $\left\{v_{n}\right\}$ has no convergent subsequence. (To wit, let $v=1_{2}$, $v_{n}=e_{n}$, where $e_{n}$ is a sequence with $n$th place equal to 1 and 0 elsewhere. Then $\left\{e_{n}\right\}$ cannot have a convergent subsequence.)

Let $u \in U$ with $\|u\|=1$. Then $\left\{u \otimes v_{n}\right\}$ is a bounded sequence in $U \otimes_{p} V$. Now, $T\left(u \otimes v_{n}\right)=T_{1} u \otimes v_{n}$ and

$$
\left\|T\left(u \otimes v_{n}\right)-T\left(u \otimes v_{m}\right)\right\|_{p}=\left\|T_{1} u \otimes v_{n}-T_{1} u \otimes v_{m}\right\|_{p}=\left\|T_{1} u\right\|\left\|v_{n}-v_{m}\right\| .
$$

Equation (2.13) shows that $\left\{T\left(u \otimes v_{n}\right)\right\}$ has a convergent subsequence only if the sequence $\left\{v_{n}\right\}$ has a convergent subsequence. This ascertains that $T$ cannot be compact. So, we are forced to consider $V$ to be finite dimensional.

(ii) Although $\operatorname{sp}(T)$ is, in general, a larger set than $\operatorname{sp}\left(T_{1}\right)$, the result $\operatorname{sp}(T)=\operatorname{sp}\left(T_{1}\right)$, indicates that many eigenvalues of $T$ repeat the same eigenvalue. For the sake of completeness, we illustrate the situation with an example. 
Let $\mathscr{A}$ be the Banach algebra of $2 \times 2$ real square matrices with $\|A\|=\max \left\{\left|u_{i}\right|: i=\right.$ $1,2,3,4\}$, where

$$
A=\left[\begin{array}{ll}
u_{1} & u_{2} \\
u_{3} & u_{4}
\end{array}\right] \in \mathscr{A}
$$

Let $U=V=\mathscr{A}$. Put

$$
e_{1}=\left[\begin{array}{ll}
1 & 0 \\
0 & 0
\end{array}\right], \quad e_{2}=\left[\begin{array}{ll}
0 & 1 \\
0 & 0
\end{array}\right], \quad e_{3}=\left[\begin{array}{ll}
0 & 0 \\
1 & 0
\end{array}\right], \quad e_{4}=\left[\begin{array}{ll}
0 & 0 \\
0 & 1
\end{array}\right] .
$$

Then $\beta=\left\{e_{1}, e_{2}, e_{3}, e_{4}\right\}$ is a basis of $\mathcal{A}$.

Define a map $T_{1}: \mathscr{A} \rightarrow \mathscr{A}$ by

$$
T_{1} A=\left[\begin{array}{cc}
\sum_{i=1}^{4} \alpha_{i} u_{i} & \sum_{i=1}^{4} \beta_{i} u_{i} \\
\sum_{i=1}^{4} \gamma_{i} u_{i} & \sum_{i=1}^{4} \delta_{i} u_{i}
\end{array}\right] .
$$

Then it is evident that $T_{1}$ is a linear operator on $\mathscr{A}$ and the matrix representation of $T_{1}$ with respect to the basis $\beta$ is

$$
\left[T_{1}\right]_{\beta}=\left[\begin{array}{llll}
\alpha_{1} & \alpha_{2} & \alpha_{3} & \alpha_{4} \\
\beta_{1} & \beta_{2} & \beta_{3} & \beta_{4} \\
\gamma_{1} & \gamma_{2} & \gamma_{3} & \gamma_{4} \\
\delta_{1} & \delta_{2} & \delta_{3} & \delta_{4}
\end{array}\right] .
$$

Next, $\left\{e_{i} \otimes e_{j}: i, j=1,2,3,4\right\}$ is a basis for $\mathscr{A} \otimes_{p} \mathscr{A}$. If we define an operator $T: \mathscr{A} \otimes_{p} \mathscr{A} \rightarrow$ $\mathscr{A} \otimes_{p} \mathscr{A}$ by $T\left(\sum_{i} u_{i} \otimes v_{i}\right)=\sum_{i}\left(T_{1} u_{i}\right) \otimes v_{i}$, then the matrix representation of $T$ is a $16 \times 16$ square matrix, that is, exhibited below:

$$
\left[\begin{array}{cccccccccccccccc}
a_{1} & 0 & 0 & 0 & a_{2} & 0 & 0 & 0 & a_{3} & 0 & 0 & 0 & a_{4} & 0 & 0 & 0 \\
0 & a_{1} & 0 & 0 & 0 & a_{2} & 0 & 0 & 0 & a_{3} & 0 & 0 & 0 & a_{4} & 0 & 0 \\
0 & 0 & a_{1} & 0 & 0 & 0 & a_{2} & 0 & 0 & 0 & a_{3} & 0 & 0 & 0 & a_{4} & 0 \\
0 & 0 & 0 & a_{1} & 0 & 0 & 0 & a_{2} & 0 & 0 & 0 & a_{3} & 0 & 0 & 0 & a_{4} \\
b_{1} & 0 & 0 & 0 & b_{2} & 0 & 0 & 0 & b_{3} & 0 & 0 & 0 & b_{4} & 0 & 0 & 0 \\
0 & b_{1} & 0 & 0 & 0 & b_{2} & 0 & 0 & 0 & b_{3} & 0 & 0 & 0 & b_{4} & 0 & 0 \\
0 & 0 & b_{1} & 0 & 0 & 0 & b_{2} & 0 & 0 & 0 & b_{3} & 0 & 0 & 0 & b_{4} & 0 \\
0 & 0 & 0 & b_{1} & 0 & 0 & 0 & b_{2} & 0 & 0 & 0 & b_{3} & 0 & 0 & 0 & b_{4} \\
c_{1} & 0 & 0 & 0 & c_{2} & 0 & 0 & 0 & c_{3} & 0 & 0 & 0 & c_{4} & 0 & 0 & 0 \\
0 & c_{1} & 0 & 0 & 0 & c_{2} & 0 & 0 & 0 & c_{3} & 0 & 0 & 0 & c_{4} & 0 & 0 \\
0 & 0 & c_{1} & 0 & 0 & 0 & c_{2} & 0 & 0 & 0 & c_{3} & 0 & 0 & 0 & c_{4} & 0 \\
0 & 0 & 0 & c_{1} & 0 & 0 & 0 & c_{2} & 0 & 0 & 0 & c_{3} & 0 & 0 & 0 & c_{4} \\
d_{1} & 0 & 0 & 0 & d_{2} & 0 & 0 & 0 & d_{3} & 0 & 0 & 0 & d_{4} & 0 & 0 & 0 \\
0 & d_{1} & 0 & 0 & 0 & d_{2} & 0 & 0 & 0 & d_{3} & 0 & 0 & 0 & d_{4} & 0 & 0 \\
0 & 0 & d_{1} & 0 & 0 & 0 & d_{2} & 0 & 0 & 0 & d_{3} & 0 & 0 & 0 & d_{4} & 0 \\
0 & 0 & 0 & d_{1} & 0 & 0 & 0 & d_{2} & 0 & 0 & 0 & d_{3} & 0 & 0 & 0 & d_{4}
\end{array}\right] .
$$


For the matrix representation of $T_{1}$ and $T$, we found that if $T_{1}$ is a Hermitian operator, that is, $T_{1}$ has a diagonal matrix representation, then so is $T$. Moreover, putting different values of $\alpha_{i}$ 's, $\beta_{i}$ 's, $\gamma_{i}$ 's, and $\delta_{1}$ 's, we can see that $T_{1}$ and $T$ have the same eigenvalues. For instance, if we substitute

$$
\begin{array}{llll}
\alpha_{1}=1, & \alpha_{2}=-1, & \alpha_{3}=2, & \alpha_{4}=-2 ; \\
\beta_{1}=-1, & \beta_{2}=2, & \beta_{3}=3, & \beta_{4}=-3 ; \\
\gamma_{1}=2, & \gamma_{2}=3, & \gamma_{3}=3, & \gamma_{4}=1 ; \\
\delta_{1}=-2, & \delta_{2}=-3, & \delta_{3}=1, & \delta_{4}=4 ;
\end{array}
$$

then the eigenvalues of $T_{1}$ are 4.456658, 6.930083, 2.234990, and -3.621735 (correct up to six decimal places). Then the eigenvalues of $T$ are also the same each repeating four times (verified by computer).

To show some applications of Theorem 2.2, we want to concentrate on the study of the Hermitian approximation property and compact analytic semigroups on $U \otimes_{p} V$. We first recall some definitions.

(I) A Banach space $X$ is said to have the Hermitian approximation property (HAP), if for each compact subset $C$ of $X$ and every $\varepsilon>0$, there is a compact Hermitian operator $H$ on $X$ such that $\|H x-x\|<\varepsilon$ for every $x \in C$, and $\|H\| \leq 1$.

To wit, the spaces $1_{2}, c_{0}$, and so forth have the HAP.

If $X$ is separable, HAP is equivalent to the existence of a sequence $\left\{h_{m}\right\}$ with $\left\|h_{m}\right\| \leq 1$, for every $m \in \mathbb{N}$ of compact Hermitian operators on $X$ such that

$$
\left\|h_{m} x-x\right\| \rightarrow 0 \text { as } m \longrightarrow \infty \forall x \in X
$$

(II) Let $S_{\alpha}=\{z \in \mathbb{C}: \operatorname{Re}(z)>0$ and $|\operatorname{Arg}(z)|<\alpha\}$ be a sector in $\mathbb{C}$, where $\alpha$ lies in $(0, \pi / 2]$. An analytic semigroup $T^{z}$ on $X$ is a family of bounded linear operators $T^{z}: X \rightarrow X$ defined for $z \in S_{\alpha}$, where $\alpha$ is fixed, satisfying the following conditions:

(i) $T^{z_{1}} T^{z_{2}}=T^{z_{1}+z_{2}}$ for all $z_{1}, z_{2}$ in $S_{\alpha}$;

(ii) $T^{z}$ is an analytic-valued function of $z \in S_{\alpha}$;

(iii) if $x \in X$ and $\varepsilon>0$, then $\lim _{z \rightarrow 0} T^{z} x=x$ provided $z$ remains within $S_{\alpha-\varepsilon}$.

We define the generator $Z$ of $T^{z}$ by $Z x=\lim _{t \uparrow 0} t^{-1}\left(T^{t} x-x\right)$ where $t>0$ and $\operatorname{Dom}(Z)$ is the set of $x$ for which the limit exists. If all $T^{z}$ are compact operators, then we call it a compact analytic semigroup on $X$. In the following, $\mathbb{C}_{+}$denotes the right-hand half plane of the complex plane, $\mathrm{KL}(X)$ the algebra of compact operators on $X$ and "-" the closure of a set. As a first shot, a little but interesting lemma is set forth.

LEMMA 2.4. If $U$ and $V$ are arbitrary separable Banach algebras, then $U \otimes_{p} V$ is also separable.

Proof. The proof is straightforward.

The principal theorem in this section is the following.

THEOREM 2.5. If $U$ and $V$ are separable Banach algebras, then the following results are true:

(i) if $U$ has the HAP, then $U \otimes_{p} V$ has the HAP, 
(ii) if there is a compact analytic semigroup

$$
z \rightarrow T_{1}^{z}: \mathbb{C}_{+} \rightarrow \mathrm{KL}(U)
$$

such that

$$
\left(T_{1}^{z} U\right)^{-}=U, \quad\left\|T_{1}^{z}\right\| \leq 1 \forall z \in \mathbb{C}_{+},
$$

then there exists a compact analytic semigroup

$$
z \longrightarrow T^{z}: \mathbb{C}_{+} \longrightarrow \mathrm{KL}\left(U \otimes_{p} V\right)
$$

having the same kind of properties, viz,

$$
\left(T^{z}\left(U \otimes_{p} V\right)\right)^{-}=U \otimes_{p} V, \quad\left\|T^{z}\right\| \leq 1 \forall z \in \mathbb{C}_{+} .
$$

Proof. (i) Let $U$ have the Hermitian approximation property. Then there exists a sequence $\left\{T_{m}^{1}\right\}$ with $\left\|T_{m}^{1}\right\| \leq 1$ for all $m \in \mathbb{N}$ of a compact Hermitian operators on $U$ such that $\left\|T_{m}^{1} u-u\right\| \rightarrow 0$ for all $u \in U$. Then as usual for every $m \in \mathbb{N}$, define

$$
T_{m}: U \otimes_{p} V \rightarrow U \otimes_{p} V \quad \text { by } T_{m}\left(\sum_{i} u_{i} \otimes v_{i}\right)=\sum_{i}\left(T_{m}^{1} u_{i}\right) \otimes v_{i} .
$$

Then by Theorem 2.2, $T_{m}$ is a compact Hermitian operator on $U \otimes_{p} V$ such that

$$
\left\|T_{m}\right\|=\left\|T_{m}^{1}\right\| \leq 1 \quad \forall m \in \mathbb{N} .
$$

Further,

$$
\begin{aligned}
\left\|T_{m}\left(\sum_{i} u_{i} \otimes v_{i}\right)-\sum_{i}\left(u_{i} \otimes v_{i}\right)\right\|_{p} & =\left\|\sum_{i}\left(T_{m}^{1} u_{i}\right) \otimes v_{i}-\sum_{i} u_{i} \otimes v_{i}\right\|_{p} \\
& =\left\|\left(T_{m}^{1} u_{i}-u_{i}\right) \otimes v_{i}\right\|_{p} \\
& \leq \sum_{i}\left\|T_{m}^{1} u_{i}-u_{i}\right\|\left\|v_{i}\right\| \\
& \longrightarrow 0 \quad \text { as } m \rightarrow \infty,
\end{aligned}
$$

(we can choose $\left\|v_{i}\right\|=1$, for all $i$ as in Lemma 2.1). Since by Lemma 2.4, $U \otimes_{p} V$ is separable, $U \otimes_{p} V$ has the HAP.

(ii) For each $z \in \mathbb{C}_{+}$, define

$$
T^{z}\left(\sum_{i} u_{i} \otimes v_{i}\right)=\sum_{i}\left(T_{1}^{z} u_{i}\right) \otimes v_{i}
$$

We first show that $T^{z}$ is an analytic semigroup.

(1) Let $z_{1}, z_{2} \in \mathbb{C}_{+}$. Then

$$
\begin{aligned}
\left(T^{z_{1}} T^{z_{2}}\right) a & =T^{z_{1}}\left(\sum_{i}\left(T_{1}^{z_{2}} u_{i}\right) \otimes v_{i}\right)=\sum_{i} T_{1}^{z_{1}}\left(T_{1}^{z_{2}} u_{i}\right) \otimes v_{i} \\
& =\sum_{i} T_{1}^{z_{1}+z_{2}} u_{i} \otimes v_{i} \quad \text { (because } T_{1} \text { is an analytic semigroup) } \\
& =T^{z_{1}+z_{2}}(a) .
\end{aligned}
$$

So, $T^{z_{1}} T^{z_{2}}=T^{z_{1}+z_{2}}$. 
(2) Let $a=\sum_{i} u_{i} \otimes v_{i} \in U \otimes_{p} V$.

Since $T_{1}^{z}$ is analytic,

$$
\begin{aligned}
& \lim _{h \rightarrow 0} \frac{T_{1}^{z+h} u_{i}-T_{1}^{z} u_{i}}{h} \quad \text { exists for every } u_{i} \text { (w.r.t. the norm on } U \text { ) } \\
& \Longrightarrow \lim _{h \rightarrow 0}\left(\frac{T_{1}^{z+h}-T_{1}^{z}}{h}\right) u_{i} \otimes v_{i} \quad \text { exists for every pair } u_{i} \in U, v_{i} \in V \\
& \left.\quad \quad \text { (w.r.t. the norm on } U \otimes_{p} V\right) \\
& \Rightarrow \sum_{i}\left[\lim _{h \rightarrow 0}\left(\frac{T_{1}^{z+h}-T_{1}^{z}}{h}\right)\right] u_{i} \otimes v_{i} \text { exists } \\
& \Longrightarrow \lim _{h \rightarrow 0}\left(\frac{T^{z+h}-T^{z}}{h}\right) a \text { exists. }
\end{aligned}
$$

So, $T^{z}$ is an analytic semigroup of $z \in \mathbb{C}_{+}$.

Also,

$$
\begin{aligned}
\lim _{z \rightarrow 0} T^{z} a & =\lim _{z \rightarrow 0} \sum_{i}\left(T_{1}^{z} u_{i}\right) \otimes v_{i}=\sum_{i}\left(\lim _{z \rightarrow 0} T_{1}^{z} u_{i}\right) \otimes v_{i} \\
& =\sum_{i}\left(u_{i} \otimes v_{i}\right) \quad \text { because } T_{1}^{z} \text { is analytic } \\
& =a .
\end{aligned}
$$

Consequently, $T^{z}$ is an analytic semigroup. By Theorem 2.2, the compactness of $T_{1}^{z}$ implies that of $T^{z}$. To show that $\left(T^{z}\left(U \otimes_{p} V\right)\right)^{-}=U \otimes_{p} V$, let $a=\sum_{j=1}^{k} u_{j} \otimes e_{j}$ by any element of $U \otimes_{p} V$ and $\varepsilon>0$. $\left(T_{1}^{z} U\right)^{-}=U$ provides an element $u_{j}^{\prime} \in U$ such that $\left\|T_{1}^{z} u_{j}^{\prime}-u_{j}\right\|<\varepsilon / k$.

Let $a^{\prime}=\sum_{j=1}^{k} u_{j}^{\prime} \otimes e_{j}$. Then

$$
\begin{aligned}
\left\|T^{z} a^{\prime}-a\right\|_{p} & =\left\|\sum_{j=1}^{k} T_{1} u_{j}^{\prime} \otimes e_{j}-\sum_{j=1}^{k} T_{1} u_{j} \otimes e_{j}\right\|_{p} \\
& \leq \sum_{j=1}^{k}\left\|T_{1} u_{j}^{\prime}-u_{j}\right\|\left\|e_{j}\right\| \\
& <\varepsilon .
\end{aligned}
$$

This guarantees that $\left(T^{z}\left(U \otimes_{p} V\right)\right)^{-}=U \otimes_{p} V$, for all $z \in \mathbb{C}_{+}$. Further, $\left\|T_{1}^{z}\right\|=\left\|T^{z}\right\|$ ensures that $\left\|T^{z}\right\| \leq 1$, for all $z \in \mathbb{C}_{+}$.

Our next goal is to study the orthogonality of the null space of $T$ with its range.

We again recall some definitions. Let $X$ be a normed linear space and $x, y \in X$. If $\|x-\lambda y\| \geq\|\lambda y\|$ for all $\lambda \in \mathbb{C}$, then $x$ is said to be orthogonal to $y$. Let $M$ and $N$ be two subspaces of $X$. If $\|m+n\| \geq\|n\|$ for all $m \in M$ and for all $n \in N$, then $M$ is said to be orthogonal to $N$, and then we write $M \perp N$ (for details, see [2]).

THEOREM 2.6. (i) If $N_{1}$ and $R_{1}$ are the null space and the range of $T_{1}$, then $N=$ $N_{1} \otimes V$ and $R=R_{1} \otimes V$ are the respective null space $N$ and the range $R$ of $T$. 
(ii) If $N_{1} \perp R_{1}$, then $N \perp R$, provided the basis $\left\{e_{j}\right\}$ is chosen in such a way that

$$
\|a\|=\left\|\sum_{j=1}^{k} u_{j} \otimes e_{j}\right\|=\sum_{j=1}^{k}\left\|u_{j}\right\| \quad \forall a \in U \otimes_{p} V .
$$

Proof. It is not so hard to establish the results.

The CONVERSE Problems. The main objective here is to investigate the possibility of studying the converse of the above results. To be precise, for a given compact Hermitian operator $T$ on $U \otimes_{p} V$, can we obtain a compact Hermitian operator $T_{1}$ on $U$ such that $\|T\|=\left\|T_{1}\right\|$ and $\operatorname{sp}(T)=\operatorname{sp}\left(T_{1}\right)$ ? Some possibilities are highlighted below. We first state a lemma whose proof is straightforward, and hence omitted.

LEMMA 2.7. For each $j(j=1,2, \ldots, k)$, let $T_{j}$ be a map from $U \otimes_{p} V$ into $U$ defined by $T_{j}(a)=u_{j}$ for every element $a=\sum_{j=1}^{k} u_{j} \otimes e_{j}$ in $U \otimes_{p} V$. Then $T_{j}$ 's are continuous linear transformation.

THEOREM 2.8. Every compact Hermitian bounded operator $T$ on $U \otimes_{p} V$ gives rise to $k^{2}$ number of linear operators $T^{i, j}(i, j=1,2, \ldots, k)$ on $U$ such that

(i) $T^{i, j}$ is compact for all $i, j$.

(ii) If $T^{i, j}=0$ for $i \neq j$, then $T^{i, j}$ is Hermitian, $\left\|T^{i, j}\right\| \leq\|T\|$ for all $j=1,2, \ldots, k$ and $\bigcup_{j=1}^{k} \operatorname{sp}\left(T^{j, j}\right)=\operatorname{sp}(T)$.

Proof. Let $T$ be a compact Hermitian operator on $U \otimes_{p} V$. For fixed $i, j(i, j=$ $1,2, \ldots, k)$, we define an operator $T^{i, j}$ as follows.

Let $u$ be an arbitrary element of $U$ and let $T\left(u \otimes e_{i}\right)=\sum_{\ell=1}^{k} u_{\ell} \otimes e_{\ell}$. Then $T^{i, j}: U \rightarrow U$ is a map defined by $T^{i, j}(u)=u_{j}$. Since the expression $\sum_{\ell=1}^{k} u_{\ell} \otimes e_{\ell}$ for every element $a \in U \otimes_{p} V$ is unique, $T^{i, j}$ is well defined.

(1) The linearity of $T^{i, j}$ is obvious.

(2) We wish to show that $T^{i, j}$ is bounded.

Now, for each $u \in U, T\left(u \otimes e_{i}\right)=\sum_{\ell=1}^{k} T^{i, \ell}(u) \otimes e_{\ell}$. For a fixed $j(j=1,2,3, \ldots, k)$, we define a map $f_{j}$ by $f_{j}: U \otimes_{p} V \rightarrow U$ and $f_{j}(a)=u_{j}$, where $a=\sum_{\ell=1}^{k} u_{\ell} \otimes e_{\ell}$. Then $f_{j}$ is a bounded linear operator by Lemma 2.7 .

So,

$$
\begin{aligned}
\left\|T^{i, j} u\right\| & =\left\|f_{j}\left(T\left(u \otimes e_{i}\right)\right)\right\| \\
& \leq\left\|f_{j}\right\|\left\|T\left(u \otimes e_{i}\right)\right\| \\
& \leq\left\|f_{j}\right\|\|T\|\left\|u \otimes e_{i}\right\|_{p} \\
& =K\|u\|, \quad \text { where } K=\left\|f_{j}\right\|\|T\| .
\end{aligned}
$$

Hence, $T^{i, j}$ is bounded.

(i) Next, our purpose is to prove the compactness of $T^{i, j}$. Let $\left\{u_{n}\right\}$ be a sequence in $U$ with $\left\|u_{n}\right\| \leq 1$, for all $n$. Then for a fixed $i(i=1,2, \ldots, k)$,

$$
\left\|u_{n} \otimes e_{i}\right\|_{p}=\left\|u_{n}\right\|\left\|e_{i}\right\|=\left\|u_{n}\right\| \leq 1 \quad \forall n .
$$

Hence for a fixed $i,\left\{u_{n} \otimes e_{i}\right\}_{n=1}^{\infty}$ is a bounded sequence in $U \otimes_{p} V$. The compactness of $T$ yields that $\left\{T\left(u_{n} \otimes e_{i}\right)\right\}$ has a convergent subsequence, say $\left\{T\left(u_{n_{m}} \otimes e_{i}\right)\right\}$ 
converging to

$$
x=\sum_{\ell=1}^{k} x_{\ell} \otimes e_{\ell} \quad \text { in } U \otimes_{p} V .
$$

Now,

$$
\begin{aligned}
& \lim _{m \rightarrow \infty}\left\|T\left(u_{n_{m}} \otimes e_{i}\right)-\sum_{\ell=1}^{k} x_{\ell} \otimes e_{\ell}\right\|_{p}=0 \\
& \Longrightarrow \lim _{m \rightarrow \infty}\left\|\sum_{\ell=1}^{k}\left(T^{i, \ell} u_{n_{m}}\right)-\sum_{\ell=1}^{k} x_{\ell} \otimes e_{\ell}\right\|_{p}=0 \\
& \Longrightarrow\left\|\sum_{\ell=1}^{k}\left[\left(\lim _{m \rightarrow \infty} T^{i, \ell} u_{n_{m}}-x_{\ell}\right) \otimes e_{\ell}\right]\right\|_{p}=0 \quad \text { because } T^{i, \ell} \text { are continuous } \\
& \Longrightarrow \sum_{\ell=1}^{k} \lim _{m \rightarrow \infty}\left(T^{i, \ell} u_{n_{m}}-x_{\ell}\right) \otimes e_{\ell}=0 .
\end{aligned}
$$

Hence $\lim _{m \rightarrow \infty} T^{i, \ell} u_{n_{m}}=x_{\ell}$, for all $\ell=1,2, \ldots, k$. So, for $\ell=j$, we have that $T^{i, j}$ is compact.

(ii) Now suppose that $T^{i, j}=0$ for $i \neq j$.

(I) We want to show that $T^{j, j}$ is Hermitian for each $j=1,2, \ldots, k$.

$T$ is Hermitian and so $\left\|e^{i \alpha T}\right\|=1$, for all $\alpha \in \mathbb{R}$. Let $u \in U$ with $\|u\|=1$. Then for fixed $j,\left\|u \otimes e_{j}\right\|_{p}=1$.

Now,

$$
\begin{aligned}
1=\left\|e^{i \alpha T}\right\| & =\sup \left\{\left\|e^{i \alpha T} a\right\|_{p}:\|a\|_{p}=1\right\} \geq\left\|e^{i \alpha T}\left(u \otimes e_{j}\right)\right\|_{p} \\
& =\left\|\left[1+i \alpha T+\frac{(i \alpha T)^{2}}{2 !}+\frac{(i \alpha T)^{3}}{3 !}+\cdots\right]\left(u \otimes e_{j}\right)\right\|_{p} \\
& =\left\|\left[u+i \alpha T^{i, j} u+\frac{\left(i \alpha T^{i, j}\right)^{2}}{2 !} u+\frac{\left(i \alpha T^{j, j}\right)^{3}}{3 !} u+\cdots\right] \otimes e_{j}\right\|_{p} \\
& =\left\|e^{i \alpha T^{j, j}} u\right\| \text { because }\left\|e_{j}\right\|=1 .
\end{aligned}
$$

Thus, $\left\|e^{i \alpha T^{j, j}} u\right\| \leq 1$, for all $u \in U$ with $\|u\|=1$. This gives $\left\|e^{i \alpha T^{j, j}}\right\| \leq 1$. Again, $e^{i \alpha T^{j, j}} e^{-i \alpha T^{j, j}}=I$, so, $1=\|I\|=\left\|e^{i \alpha T^{j, j}} e^{-i \alpha T^{j, j}}\right\|_{p} \leq\left\|e^{i \alpha T^{j, j}}\right\|_{p}\left\|e^{-i \alpha T^{j, j}}\right\|_{p}$.

This yields $\left\|e^{-i \alpha T^{j, j}}\right\| \geq 1 /\left\|e^{i \alpha T^{j, j}}\right\| \geq 1$. Since $\alpha \in \mathbb{R}$ then $-\alpha \in \mathbb{R}$, we can obtain $\left\|e^{i \alpha T^{j, j}}\right\| \geq 1$ and hence $\left\|e^{i \alpha T^{j, j}}\right\|=1$. This implies that $T^{j, j}$ is Hermitian.

(II) Again for fixed $j$,

$$
\left\|u \otimes e_{j}\right\|_{p}=1, \quad \text { whenever } u \in U,\|u\|=1 .
$$

Now, $T\left(u \otimes e_{j}\right)=\left(T^{j, j} u\right) \otimes e_{j}$. So, $\|T\| \geq\left\|T\left(u \otimes e_{j}\right)\right\|_{p}=\left\|T^{j, j}\left(u \otimes e_{j}\right)\right\|_{p}=\left\|T^{j, j} u\right\|$. This gives $\left\|T^{j, j}\right\| \leq\|T\|, j=1,2, \ldots, k$.

(III) We wish to show that $\bigcup_{j=1}^{k} \operatorname{sp}\left(T^{j, j}\right)=\operatorname{sp}(T)$. 
Let

$$
\begin{aligned}
\lambda \in \bigcup_{j=1}^{k} \operatorname{sp}\left(T^{j, j}\right) & \Longrightarrow \lambda \in \operatorname{sp}\left(T^{j, j}\right) \quad \text { for some } j \\
& \Longrightarrow\left(T^{j, j}-\lambda I\right) \quad \text { is singular } \\
& \Longrightarrow \text { there exists a nonzero vector } u \in U \text { such that }\left(T^{j, j}-\lambda I\right) u=0 \\
& \Longrightarrow\left(T^{j, j}-\lambda I\right) u \otimes e_{j}=0 \\
& \Longrightarrow(T-\lambda I) u \otimes e_{j}=0 \quad \text { because } u \neq 0, e_{j} \neq 0 \Longrightarrow u \otimes e_{j} \neq 0 \\
& \Longrightarrow \lambda \in \operatorname{sp}(T) .
\end{aligned}
$$

This gives $\bigcup_{j=1}^{k} \operatorname{sp}\left(T^{j, j}\right) \subseteq \operatorname{sp}(T)$.

On the other hand,

$$
\begin{aligned}
\lambda \in \operatorname{sp}(T) & \Longrightarrow T-\lambda I \text { is singular } \\
& \Longrightarrow \exists a \text { nonzero vector } a=\sum_{\ell=1}^{k} u_{\ell} \otimes e_{\ell} \text { such that }(T-\lambda I) a=0 \\
& \Longrightarrow \sum_{\ell=1}^{k} T\left(u_{\ell} \otimes e_{\ell}\right)-\lambda \sum_{\ell=1}^{k} u_{\ell} \otimes e_{\ell}=0 \\
& \Longrightarrow \sum_{\ell=1}^{k} T^{\ell, \ell} u_{\ell} \otimes e_{\ell}-\lambda \sum_{\ell=1}^{k} u_{\ell} \otimes e_{\ell}=0 \\
& \Longrightarrow \sum_{\ell=1}^{k}\left(T^{\ell, \ell}-\lambda I\right) u_{\ell} \otimes e_{\ell}=0 \\
& \Longrightarrow\left(T^{\ell, \ell}-\lambda I\right) u_{\ell}=0 ; \quad \forall \ell=1,2, \ldots, k
\end{aligned}
$$

Since $a \neq 0$, there exists at least one $j$ such that $u_{j} \neq 0$. For this $u_{j}$, we have

$$
\begin{aligned}
\left(T^{j, j}-\lambda I\right) u_{j}=0 & \Longrightarrow \lambda \in \operatorname{sp}\left(T^{j, j}\right) \\
& \Longrightarrow \lambda \in \bigcup_{j=1}^{k} \operatorname{sp}\left(T^{j, j}\right) .
\end{aligned}
$$

Thus,

$$
\bigcup_{j=1}^{k} \operatorname{sp}\left(T^{j, j}\right)=\operatorname{sp}(T) .
$$

This completes the proof of the theorem.

We are now in a position to pose some open problems.

(i) Does the HAP in $U \otimes_{p} V$ give the existence of the HAP in $U$ ?

(ii) If the null space of $T$ is orthogonal to its range, is the null space $T^{i, j}$ orthogonal to its range? What are the orthogonal complements of the null space and the range of $T$ ? 
(iii) Suppose that the operator $T$ is the derivation (for definition of a derivation, see [5]). Is $T^{i, j}$ a derivation?

(iv) Can we make the analogous study in case of other tensor products, viz, the injective tensor products, the Haagerup tensor product, and so forth, [1]?

\section{REFERENCES}

[1] S. D. Allen, A. M. Sinclair, and R. R. Smith, The ideal structure of the Haagerup tensor product of $C^{*}$-algebras, J. Reine Angew. Math. 442 (1993), 111-148.

[2] J. Anderson, On normal derivations, Proc. Amer. Math. Soc. 38 (1973), 135-140.

[3] D. P. Blecher, Geometry of the tensor product of $C^{*}$-algebras, Math. Proc. Cambridge Philos. Soc. 104 (1988), no. 1, 119-127.

[4] B. Bollobás, The spectral decomposition of compact hermitian operators on Banach spaces, Bull. London Math. Soc. 5 (1973), 29-36.

[5] F. F. Bonsall and J. Duncan, Numerical Ranges. II, London Mathematical Society Lecture Notes Series, no. 10, Cambridge University Press, London, 1973.

[6] T. K. Carne, Tensor products and Banach algebras, J. London Math. Soc. (2) 17 (1978), no. 3, 480-488.

[7] T. K. Dutta, Hermitian elements in projective tensor products, Indian J. Pure Appl. Math. 21 (1990), no. 7, 605-616.

T. K. DutTA, H. K. NATH, AND H. K. SARMAh: DEPARTMENT OF MATHEMATICS, GAUHATI UNIVERSITY, GUWAHATI-781014, ASSAM, INDIA 


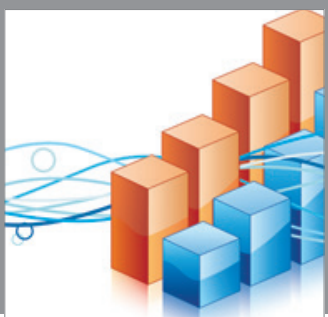

Advances in

Operations Research

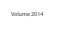

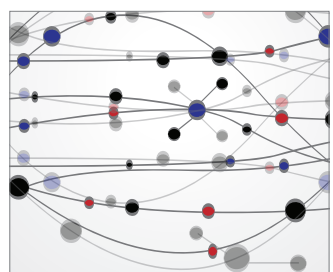

\section{The Scientific} World Journal
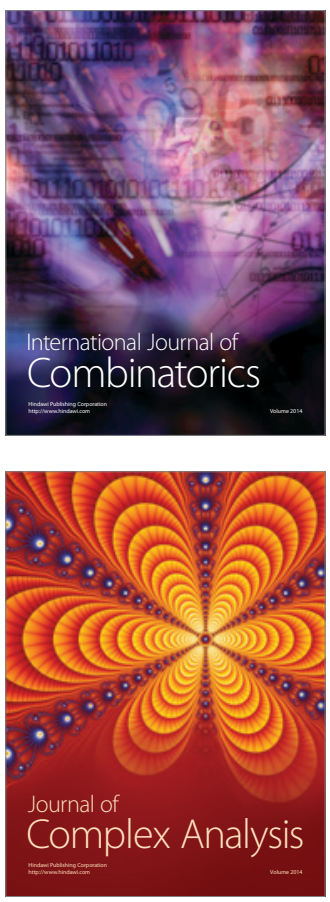

International Journal of

Mathematics and

Mathematical

Sciences
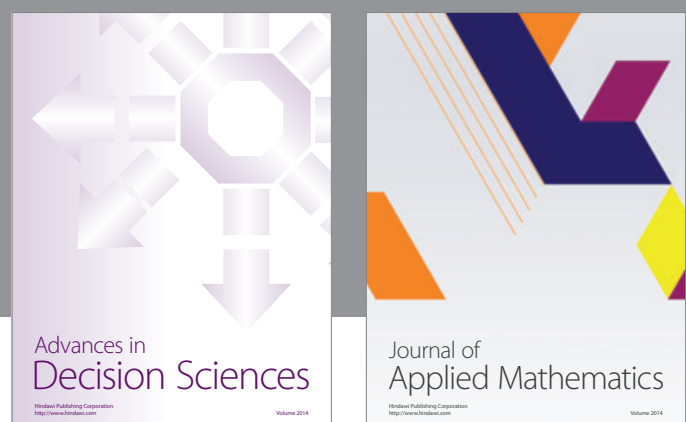

Journal of

Applied Mathematics
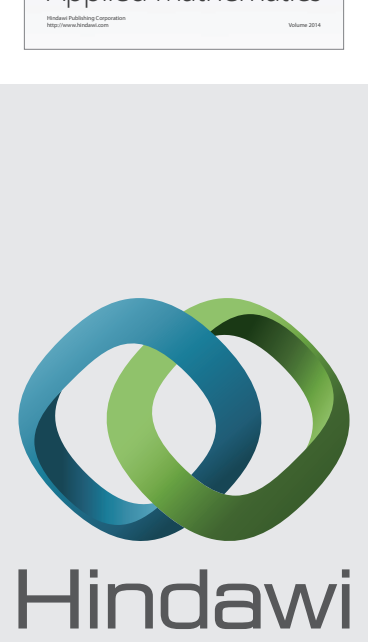

Submit your manuscripts at http://www.hindawi.com
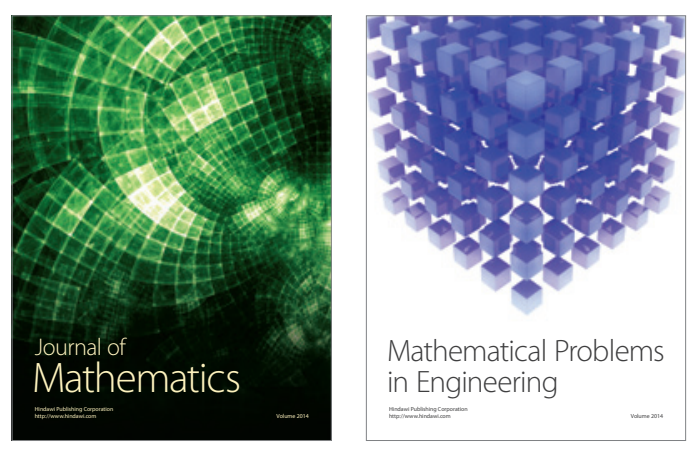

Mathematical Problems in Engineering
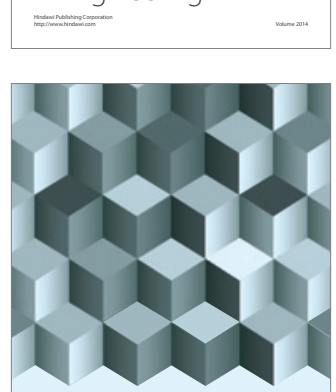

Journal of

Function Spaces
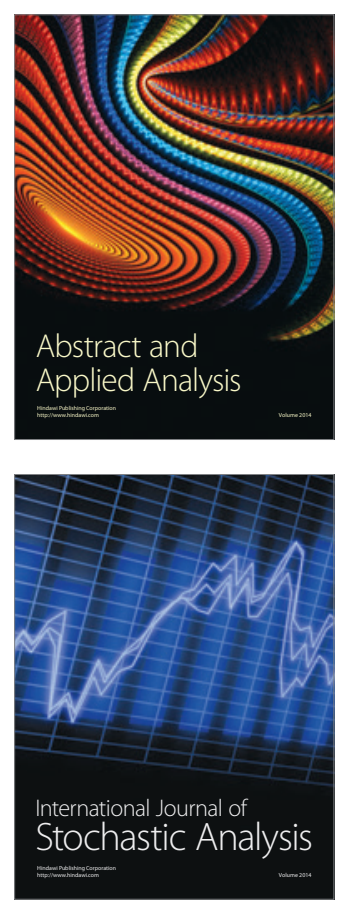

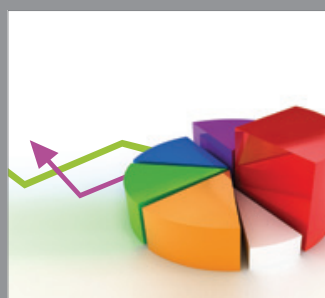

ournal of

Probability and Statistics

Promensencen
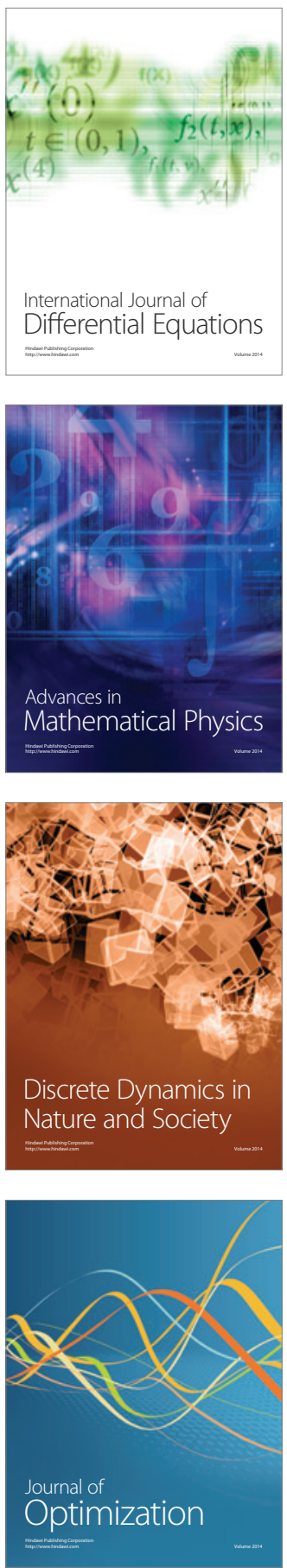